Note

\section{Recovery of Less-volatile Chemicals from Pure Fat Using a Simultaneous Steam Distillation-extraction Apparatus}

\author{
Fernando E. Figuerola* \\ and Takayuki SHIBAmoto \\ Department of Environmental Toxicology, \\ University of California, Davis, \\ Davis, CA 95616, U.S.A.
}

Received May 19, 1983

Multi-residue analysis has been one of the most difficult problems to solve in residue analysis. Many methods have been developed for isolating a single pesticide. ${ }^{1 \sim 5)}$ They are, however, complex and tedious procedures and are not applicable for multi-residue analysis.

A unique apparatus (Likens-Nickerson apparatus)

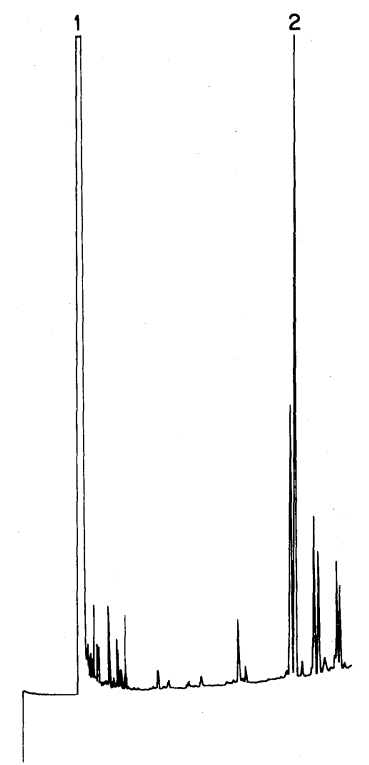

FIG. 1. A Gas Chromatogram of a Dichloromethane Extract Obtained from an Unspiked Fat Sample (Blank) on a WCOT Glass Capillary Column $(15 \mathrm{~m} \times 0.28 \mathrm{~mm}$ i.d. $)$ Coated with OV-101.

Column held at $180^{\circ} \mathrm{C}$ for $8 \mathrm{~min}$, programmed at $1^{\circ} \mathrm{C} / \mathrm{min}$ to $230^{\circ} \mathrm{C}$ and held. Inlet and detector were maintained at $250^{\circ} \mathrm{C}$; the linear herium carrier gas flow rate was $22 \mathrm{~cm} / \mathrm{sec}$. Peak $\# 1$ = solvent; peak $\# 2=$ internal standard. which allows simultaneous steam distillation and organic solvent extraction was invented in 1966. ${ }^{6)}$ Since then, many volatile samples have been prepared using this apparatus. ${ }^{79)}$ There are, however, virtually no reports on the application of this apparatus for analysis of lessvolatile chemicals such as pesticides.

The use of a high resolution glass capillary column is excellent for qualitative and quantitative analysis of multiple components. ${ }^{10)}$ However, despite the advantages of open tubular columns, there are only a few reports published on their use in pesticide analysis, and almost all the routine gas chromatographic analyses of these substances are still performed with packed columns.

In this study, less-volatile multi-residue analysis of pure fat samples using a simultaneous steam distillation-solvent extraction apparatus and a glass capillary gas chromatographic column was investigated.

The test system consisted of $1000 \mathrm{ml}$ of water plus $40 \mathrm{~g}$ of pure fat obtained from the renal periphery of a beef carcass. The mixture was spiked with $10 \mathrm{ppm}$ each of sevin, heptachlor, parathion and aldrin, and subjected to simultaneous steam distillation-solvent (dichloromethane) extraction. Four different extraction periods $(20,25,30$, $35 \mathrm{hr}$ ) were used to investigate the effect of the length of extraction on the recovery efficiency.

The recovery efficiency was determined using the gas

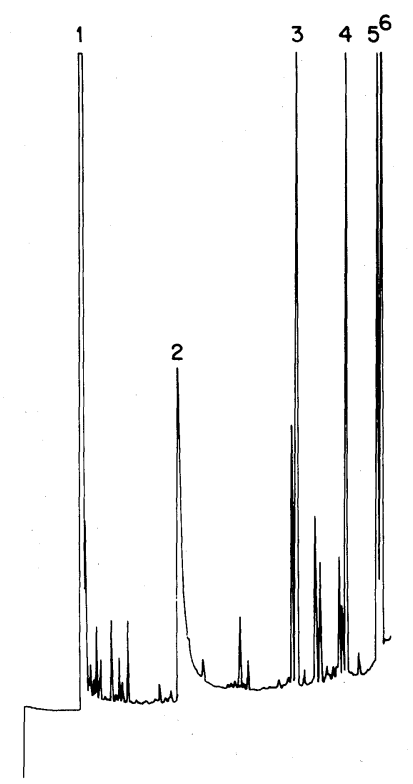

Fig. 2. A Typical Gas Chromatogram of a Dichloromethane Extract Obtained from the Pesticides Spiked Fat Sample.

Same conditions as in Fig. 1. Peak $\# 1=$ solvent; peak $\# 2=$ sevin; peak $\# 3=$ internal standard; peak $\# 4=$ heptachlor; peak $\# 5=$ parathion; peak $\# 6=$ aldrin.

\footnotetext{
* Present address: Department of Food Science and Technology, University of Chile, Santiago, Chile.
} 
Table I. Recovery Efficiencies for the Pesticides at Different Extraction Times

\begin{tabular}{lllll}
\hline Pesticide & $20 \mathrm{hr}$ & $25 \mathrm{hr}$ & $30 \mathrm{hr}$ & $35 \mathrm{hr}$ \\
\hline Sevin & $49.7 \pm 6.7^{a}$ & $52.6 \pm 1.05$ & $53.5 \pm 6.9$ & $58.2 \pm 4.8$ \\
Heptachlor & $40.7 \pm 6.8$ & $42.3 \pm 4.9$ & $49.0 \pm 3.7$ & $46.9 \pm 4.1$ \\
Parathion & $31.2 \pm 6.8$ & $33.9 \pm 5.5$ & $38.9 \pm 4.1$ & $42.4 \pm 4.9$ \\
Aldrin & $43.7 \pm 7.0$ & $42.8 \pm 4.6$ & $53.0 \pm 5.1$ & $57.6 \pm 7.3$ \\
\hline
\end{tabular}

${ }^{a}$ (Amount of pesticide recovered/amount of pesticide spiked) $\times 100$. Each value is the average of five determinations followed by the standard deviation.

chromatographic internal standard method with octadecane as internal standard. ${ }^{11)}$ A Hewlett-Packard Model 5880A gas chromatograph equipped with a flame ionization detector and a glass capillary column $(15 \mathrm{~m} \times$ $0.28 \mathrm{~mm}$ i.d.) coated with OV-101 was used. In order to assure the identity of the peaks in the chromatogram, the Kovats Indices for the four pesticides were determined on an $\mathrm{OV}-101$ column at $200^{\circ} \mathrm{C}$ according to the method described previously. ${ }^{10}$ ) The Kovats Indices for the standard pesticides on OV-101 are as follows: sevin $=1551$, heptachlor $=1907$, parathion $=1964$, and aldrin $=1972$.

Figure 1 shows a chromatogram of a blank sample obtained from an unspiked fat sample with simultaneous steam distillation extraction for $35 \mathrm{hr}$. In the chromatogram, all the peaks, except peak \#1 (solvent dichloromethane) and peak \#2 (octadecane, internal standard), correspond to substances produced by heating fat. Figure 2 shows a typical chromatogram of a spiked sample extract. Comparison of the two figures indicates that interfering peaks at the retention times where the pesticides eluted from the column are negligible. The two chromatograms indicate that neither the process nor the chromatographic analysis produced significant changes in the pesticides.

The results for the recovery efficiencies in terms of the means \pm standard deviation for five determinations are presented in Table I. The recovery efficiencies for the four pesticides ranged from $31.2 \%$ to $58 \%$ for parathion with $20 \mathrm{hr}$ extraction and sevin with $35 \mathrm{hr}$ extraction, respectively. The recovery efficiencies were not high for all pesticides used. The low recovery may be due to the high content of pure fat. On the other hand, reproducibility of recovery was excellent.

The quantitative analysis of less-volatile chemicals may be performed with this apparatus if the recovery efficiency is established and is reproducible. This apparatus might be more useful in isolation of less-volatile compounds from high lipid content samples for qualitative analysis rather than for quantitative analysis. The simplicity may compensate for yield in the recovery if the analytical object is not quantitative. A glass capillary column showed excellent resolution in multi-residue analysis.

\section{REFERENCES}

1) K. Hammarstrand, "Gas Chromatographic Analysis of Pesticides," Varian Associates, Palo Alto, 1976, p. 96.

2) R. A. Flath, R. R. Forrey and D. G. Guadagni, J. Agric. Food Chem., 21, 948 (1973).

3) W. W. Thornburg, "Analytical Methods for Pesticides, Plant Growth Regulators and Food Additives," ed. by G. Zweig, Vol. I, Academic Press, New York, 1963, p. 87.

4) M. G. Clear, F. R. Fowler, S. R. B. Solly and A. R. Pitchie, New Zealand J. Sci., 20, 221 (1977).

5) H. A. Stansbury, Jr. and R. Miskus, "Analytical Methods for Pesticides, Plant Growth Regulators and Food Additives," Vol. II, ed. by G. Zweig, Academic Press, New York, 1964, p. 321.

6) S. T. Likens and G. G. Nickerson, J. Chromatogr., 21, 1 (1966).

7) H. Maase and R. E. Kepner, J. Agric. Food Chem., 18, 1095 (1970).

8) T. H. Schultz, D. R. Black, T. R. Mon and G. E. Connolly, J. Agric. Food Chem., 24, 862 (1976).

9) K. Yamaguchi and T. Shibamoto, J. Agric. Food Chem., 27, 847 (1979).

10) W. G. Jennings, "Gas Chromatography with Glass Capillary Columns," Academic Press, New York, 1980.

11) L. S. Ettre, Interpretation of Analytical Results, "The Practice of Gas Chromatography," ed. by L. S. Ettre and A. Zlatkis, Interscience, New York, 1967, p. 381 . 